\title{
Representación de la enfermedad, ajuste psicosocial y malestar psicológico en pacientes con un trastorno de la conducta alimentaria
}

\section{IIIness representation, psychosocial adjustment and psychological distress in patients with eating disorders}

Fecha de recepción: 05-07-2018

Fecha de aceptación: 15-07-2018
Marta Escolano Herrera

Centro CREA. Universidad Miguel Hernández

Yolanda Quiles Marcos

Departamento Psicología de la Salud

Universidad Miguel Hernández

Cebtro CREA

María José Quiles Sebastián

Departamento Psicología de la Salud. Universidad Miguel Hernández.

Centro CREA

Lidia Pamies Aubalat

Universidad Católica de Murcia

\section{resumen/alostract:}

El presente trabajo tuvo como objetivo describir la representación de la enfermedad en pacientes con un trastorno de la conducta alimentaria (TCA) y evaluar su relación con el ajuste psicosocial y el malestar psicológico, siguiendo el Modelo de Auto-Regulación de Leventhal. Para ello, participaron 56 pacientes diagnosticados de un TCA, que estaban siendo atendidos en una unidad especializada y en un centro de Atención Primaria, y cumplimentaron el BIPQ, el PAISSR, el HADS y el GHQ-12. Los pacientes percibieron que su enfermedad tenía consecuencias serias para su vida y que el tratamiento ayudaría a mejorar su condición. Consideraron que el trastorno tenía emociones negativas asociadas, además de poseer una percepción de escaso control personal sobre el problema. La percepción de consecuencias y la representación emocional se asociaron con un peor ajuste psicosocial y con el malestar psicológico. Sin embargo, la percepción de control personal se relacionó con un mejor ajuste psicosocial y emocional. Los hallazgos de este trabajo señalan la importancia de considerar las cogniciones individuales de estos pacientes sobre su enfermedad, con el fin de intervenir desde el tratamiento sobre estas creencias para mejorar el ajuste y disminuir las repercusiones emocionales negativas.

The present work had as objective describing the illness representation in patients with eating disorders (ED) and evaluating its relationship with psychosocial adjustment and psychological distress following Leventhal's SelfRegulation Model. Fifty-six patients diagnosed with an ED participated, who were being treated at a specialized unit and at a primary care center, and who had completed the BIPQ, PAIS-SR, HADS, and the GHQ-12. The patients perceived that their disorder posed serious consequences to their life, and that treatment would help improve their condition. They considered that the disorder had negative emotions associated with it, in addition to them perceiving scarce control over the problem. The perception of consequences and the emotional representation were associated with worse psychosocial adjustment and psychological distress. However, the perception of personal control was related with better psychosocial and emotional adjustment. The findings in this work point out the importance of considering individual cognitions in these patients regarding their disorder, with the aim of intervening via treatment of these beliefs in order to improve the adjustment and diminish negative emotional repercussions. 


\section{palabras clave/keywords:}

Trastornos de la conducta alimentaria; representación de la enfermedad; ajuste psicosocial; malestar psicológico.

Eating disorders; illness representation; psychosocial adjustment; psychological distress.

\section{Introducción}

Un objetivo importante en Psicología de la Salud consiste en dilucidar y comprender las variables que influyen en el comportamiento humano en relación a la salud y a la enfermedad, y diseñar estrategias de intervención efectivas en base a este conocimiento (GodoyIzquierdo, López-Chicheri, López-Torrecillas, Vélez y Godoy, 2007). En esta línea, las cogniciones, tales como las creencias, las atribuciones, los pensamientos y las actitudes, ejercen un papel importante en el ajuste a los problemas de salud y en las reacciones emocionales asociadas (Sensky, 1990). Uno de los modelos teóricos que inciden en la influencia de los factores cognitivos en el desarrollo y resultados en salud en enfermedades crónicas es el Modelo de Auto-Regulación propuesto por Leventhal, Meyer y Nerenz (1980). En concreto, el modelo explica cómo las personas son entes activos que elaboran representaciones subjetivas de su enfermedad a través de la información disponible de diversas fuentes, tanto internas como externas, en relación a su condición. Dichas representaciones guían las diferentes estrategias de afrontamiento que pone en marcha el sujeto, lo que determina los resultados que obtiene en términos comportamentales, psicológicos y médicos. Estos resultados son autoevaluados posteriormente para comparar la efectividad de las estrategias de afrontamiento adoptadas y las consecuencias esperadas. En función de la valoración, la persona puede modificar la representación de su enfermedad y/o la respuesta de afrontamiento, por lo que se produce una continua retroalimentación en el circuito en la búsqueda del sentido del problema y su manejo. Además, se propone la existencia de una ruta directa entre la representación de la enfermedad y los resultados obtenidos en salud.

En el Modelo de Auto-Regulación, la representación de la enfermedad es tanto cognitiva como emocional, siendo ambos niveles de procesamiento paralelos y mutuamente interactivos (Leventhal, Diefenbach y Leventhal, 1992). La representación cognitiva está constituida por las creencias que tiene el individuo sobre su enfermedad y se ordena en torno a cuatro dimensiones -causa, consecuencias, identidad y duración-, si bien trabajos posteriores introducen otras nuevas: el control, la curación, la duración cíclica y la coherencia (Lau y Hartman, 1983; Moss-Morris et al., 2002). La causa hace referencia a los factores percibidos como generadores del problema de salud. Las consecuencias de la enfermedad aluden al impacto que ésta produce en la calidad de vida de la persona o en cómo puede influir sobre su capacidad funcional. La dimensión identidad se relaciona con la etiqueta que el sujeto pone a su enfermedad y a las nociones que posee sobre sus síntomas. La duración se refiere a las creencias sobre el curso y la posible duración del problema de salud. Las dimensiones control y curación conciernen, respectivamente, al grado de control percibido sobre la enfermedad y a las creencias sobre la eficacia del tratamiento. La duración cíclica se refiere a la creencia de que la enfermedad será variable en su evolución, con mejoras y 
empeoramientos. Por último, la coherencia corresponde al grado en que la representación que la persona construye sobre su enfermedad le permite entenderla. Por otro lado, la representación emocional hace referencia a las reacciones emocionales asociadas al problema.

Figura 1.- Modelo de Auto-Regulación (Leventhal, Meyer y Nerenz, 1980)

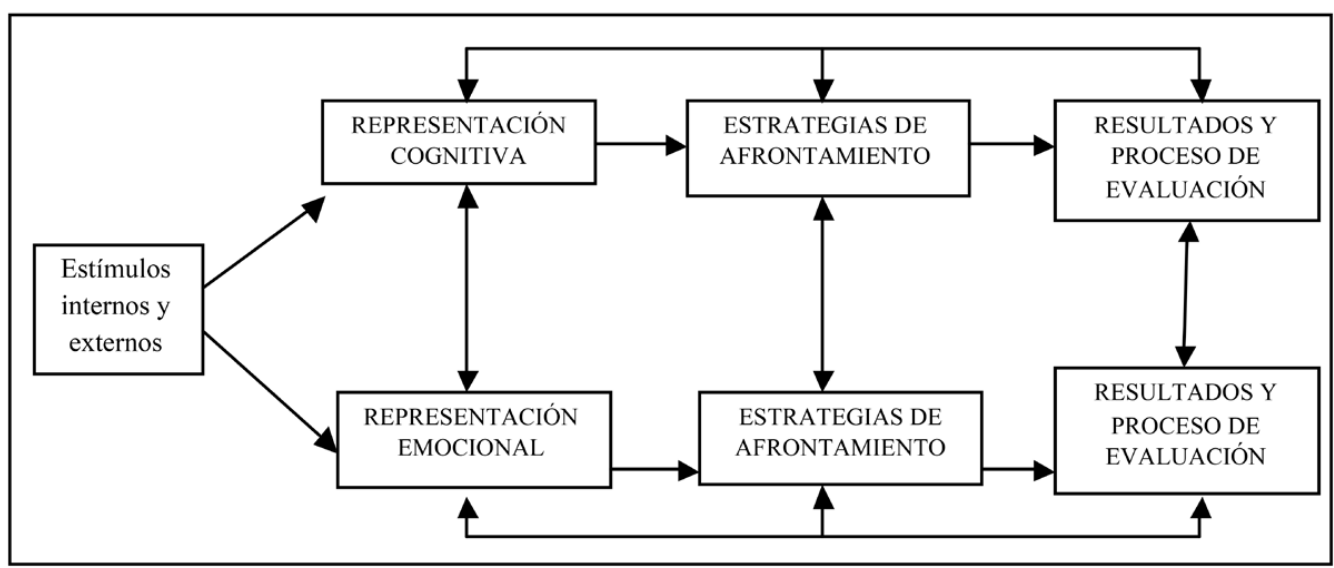

La literatura previa muestra los patrones relacionales entre las dimensiones de la representación. De este modo, la identidad se relaciona en sentido negativo con las dimensiones control y curación, y en sentido positivo con la duración y las consecuencias. Esto es, las personas que perciban una fuerte identidad con la enfermedad, creerán con mayor probabilidad que es poco susceptible de ser manejada por el control personal o por el tratamiento, crónica y con notables consecuencias para su funcionamiento. Por su parte, la dimensión control se relaciona negativamente con la percepción de duración del problema y con las consecuencias. Así, aquellos que consideren tener un control personal elevado sobre su situación, no la considerarán como crónica o de graves repercusiones (Hagger y Orbell, 2003).

El Modelo de Auto-Regulación se ha empleado también para explicar los resultados en salud, tales como el ajuste psicosocial y el estado emocional, en numerosos estudios relacionados con afecciones físicas tales como la enfermedad tiroidea autoinmune, el síndrome de fatiga crónica o la enfermedad de Parkinson, entre otros (Dickson, Toft y O'Carroll, 2009; Evans y Norman, 2009). En términos generales, la literatura indica la asociación entre resultados en salud adaptativos y una percepción del problema como no crónico, sin consecuencias graves, con baja identificación de síntomas (escasa identidad), alto control personal sobre la situación y creencia de que la enfermedad es susceptible de ser tratada de forma efectiva (Hagger y Orbell, 2003).

Existe un interés reciente por el estudio de las creencias desde esta perspectiva en problemas de salud mental como, por ejemplo, en depresión, demencia y trastornos alimentarios (Fortune, Barrowclough y Lobban, 2004; Harman y Clare, 2006; Quiles y Terol, 2011). En 
el caso de los trastornos de la conducta alimentaria (TCA), que constituyen síndromes de diferente significación clínica caracterizados por alteraciones del comportamiento respecto a la alimentación, estudios recientes han analizado diversas características cognitivas presentes en personas afectadas, tales como la percepción de control (Sassaroli, Gallucci y Ruggiero, 2008), la flexibilidad cognitiva (Roberts, Tchanturia y Treasure, 2010) o la rumiación (Cowdrey y Park, 2012). Sin embargo, las investigaciones que examinan el rol de las creencias desde la perspectiva del Modelo de Auto-Regulación en estos trastornos son escasas, a pesar de que existe evidencia de que los procesos psicológicos pueden contribuir al mantenimiento del problema (Quiles y Terol, 2011).

El primer estudio al respecto (Holliday, Wall, Treasure y Weinman, 2005) mostró que las pacientes tendieron a encontrar una fuerte identidad con el trastorno, además de percibirlo como crónico y vinculado a importantes consecuencias negativas. El trabajo reveló una percepción baja de control personal sobre la enfermedad y de la eficacia del tratamiento, dimensiones que correlacionaron negativamente con las creencias respecto a su duración. Así, las personas que percibían su problema como crónico creían poseer menos control sobre ella y que era menos tratable. Resultados similares fueron señalados por Stockford, Turner y Cooper (2007) en un trabajo sobre la percepción de la enfermedad y su relación con la motivación al cambio. En él, la etapa de precontemplación se asoció a una representación emocional baja, mientras que en la de contemplación, las consecuencias emocionales cobraron mayor importancia. La etapa de acción se relacionó con la percepción de consecuencias serias derivadas de la enfermedad y la de mantenimiento con la creencia de que el tratamiento estaba siendo eficaz.

Por su parte, Quiles, Terol, Romero y Pagán (2007) encontraron en su estudio que las pacientes de TCA, pese a percibir su enfermedad como crónica y de graves consecuencias, no mostraban una elevada identidad con ella. Además, consideraban que tenían un alto control personal sobre el problema y que éste era susceptible de mejorar mediante el tratamiento. Como señalan los autores, esta diferencia en los resultados respecto al estudio de Holliday et al. (2005) podría deberse a que todas las personas afectadas estaban recibiendo tratamiento en una unidad hospitalaria especializada en TCA, desde un enfoque cognitivo-conductual, lo cual pudo incrementar su percepción de control y sus expectativas de curación. Además, la atribución del problema a causas internas se relacionó en sentido positivo con emociones negativas, como la preocupación y el miedo. Resultados similares se hallaron en un trabajo posterior también llevado a cabo con pacientes de bulimia nerviosa (DeJong, Hillcoat, Perkins, Grover y Schmidt, 2012), en el que éstos consideraban que su enfermedad tendría mejoras y empeoramientos periódicos. También percibían su problema como crónico y con consecuencias serias, además de presentar altos niveles de ansiedad y depresión asociados.

En referencia a la relación de las creencias sobre la enfermedad con los resultados en salud en los TCA, sólo el estudio de Quiles et al. (2007) señala datos al respecto. En este trabajo se halló que la representación emocional presentó relaciones positivas y significativas con la ansiedad y la depresión. Por otro lado, las creencias de la efectividad del tratamiento y la percepción de control personal se asociaron a un mejor ajuste global, mientras que una alta identidad con la enfermedad y la atribución de la causa a factores psicológicos se relacionaron con un peor ajuste psicosocial. 
Dada la escasez de estudios referidos a la relación de las creencias sobre la enfermedad con el ajuste psicosocial y el malestar psicológico en pacientes con un TCA, y debido a la importancia que pueden tener estos resultados en el diseño de tratamientos eficaces para estos trastornos, los objetivos del presente trabajo fueron describir la representación de la enfermedad en personas afectadas por un TCA y las relaciones entre sus dimensiones, y evaluar la relación de las distintas dimensiones con el ajuste psicosocial y emocional, definido este último en términos de malestar psicológico. Para ello, se empleó un diseño transversal, descriptivo y correlacional.

Las hipótesis de partida se deducen de la literatura previa. Respecto a las relaciones entre las dimensiones de la representación de la enfermedad, se espera que: (a) la identidad presentará relaciones positivas y significativas con la percepción de duración y con las consecuencias de la enfermedad; y (b) la identidad presentará relaciones negativas y significativas con la percepción de control y de curación. En lo concerniente a la relación entre las dimensiones de la representación y el ajuste psicosocial y emocional, se espera que: (c) la identidad, la duración y las consecuencias percibidas de la enfermedad se relacionarán en negativo con el ajuste psicosocial, y en positivo con el malestar psicológico, mientras que el control y la curación lo harán en sentido positivo con el ajuste psicosocial, y en sentido negativo con el malestar psicológico.

\section{Método}

\section{Participantes}

Se reclutó un total de 56 pacientes de TCA que estaban siendo tratados en la Unidad de Trastornos Alimentarios del Hospital Universitario de San Juan, así como en una Unidad de Salud Mental de atención primaria, ambos centros en España. Un 96.4\% eran mujeres $(n=54)$ y un $3.6 \%$ eran hombres $(n=2)$. Los pacientes habían sido diagnosticados por una psiquiatra y una psicóloga de la Unidad, o por profesionales del mismo tipo pertenecientes a atención primaria, según los criterios del DSM-IV, de anorexia nerviosa (AN) (tipo restrictivo, $n=34$; tipo purgativo, $n=6$ ), bulimia nerviosa (BN) (tipo purgativo, $n=6$; tipo no purgativo, $n=3)$ o TCA no especificado (TCANE) $(n=7)$. El rango de edad de los afectados se situó entre los 12 y los 48 años $(M=21.43$; $d t=8.71)$, y la duración del trastorno se hallaba entre los 4 y los 468 meses (39 años) $(M=79.11$ meses; $d t=97.43)$. Un $45.5 \%$ de los pacientes se encontraban entre los cuatro meses y los dos años de evolución del problema, un $21.8 \%$ de ellos entre los dos y cinco años, un $12.7 \%$ entre los cinco y los 10 años, y el $20 \%$ restante de los pacientes tenía una evolución de la enfermedad de más de 10 años. Las características sociodemográficas se describen en la Tabla 1.

\section{Instrumentos}

\section{Datos sociodemográficos}

Se empleó un cuestionario creado ad-hoc para la recogida de datos sociodemográficos y características clínicas de los pacientes. Con él se obtuvo información sobre la edad, el nivel de estudios, la situación profesional, el estado civil y otros datos clínicos (diagnóstico, duración del problema y si había permanecido en ingreso hospitalario alguna vez). 
Tabla 1.- Datos sociodemográficos de las personas con el TCA

\begin{tabular}{lcc}
\hline & $\mathrm{N}$ & Porcentaje \\
\hline Nivel de estudios & 4 & \\
Estudios primarios & 23 & $7.1 \%$ \\
Graduado escolar/ESO & 7 & $41.1 \%$ \\
Bachillerato & 1 & $12.5 \%$ \\
COU & 7 & $1.8 \%$ \\
Formación profesional & 14 & $12.5 \%$ \\
Estudios universitarios & & $25 \%$ \\
\hline Situación profesional & 8 & \\
\hline Trabaja & 8 & $14.3 \%$ \\
En desempleo & 35 & $14.3 \%$ \\
Estudiante & 2 & $62.5 \%$ \\
Ama de casa & 3 & $3.6 \%$ \\
Otro & & $5.4 \%$ \\
\hline Estado civil & 8 & \\
\hline Casado/vive en pareja & 46 & $14.3 \%$ \\
Soltero & 33 & $82.1 \%$ \\
$\quad$ Con pareja & 15 & $31.3 \%$ \\
$\quad$ Sin pareja & 2 & $68.8 \%$ \\
Separado/Divorciado & & $3.6 \%$ \\
\hline Ha requerido ingreso hospitalario & 40 & $28.6 \%$ \\
\hline Sí & 16 & \\
No & & \\
\hline
\end{tabular}

\section{Percepción de la enfermedad}

Se utilizó el Brief Illness Perception Questionnaire (BIPQ; Broadbent, Petrie, Main y Weinman, 2006) en su versión española (Pacheco-Huergo, 2007). El BIPQ es un cuestionario autoinformado, compuesto por nueve escalas de ítem único que evalúan la representación de la enfermedad en las dimensiones consecuencias, duración, control, curación, identidad, coherencia, representación emocional, preocupación y causas. Para las ocho primeras dimensiones, las seleccionadas para el presente estudio, los ítems tienen una escala de respuesta tipo Likert que va de 0 a 10. Respecto a la interpretación, a mayor puntuación, mayor significación de la dimensión correspondiente. El BIPQ se ha empleado en la evaluación de la representación en diferentes trastornos mentales, como los trastornos de personalidad y 
los psicóticos (Broadbent, Kydd, Sanders, Vanderpyl, 2008), mostrando adecuadas propiedades psicométricas (Pacheco-Huergo, 2007). En este estudio, la consistencia interna fue de .77 .

\section{Ajuste psicosocial}

Para evaluar el ajuste psicosocial de los pacientes se utilizó la Psychosocial Adjustment to Illness Scale en su versión auto-informada y en español (PAIS-SR; Derogatis y López, 1983; Neipp, 2005). Se emplearon en concreto las subescalas de ajuste en el ámbito profesional/académico, ámbito doméstico, relaciones sexuales (aplicada únicamente a pacientes de 16 años en adelante), relaciones familiares y ámbito social. Estas subescalas suman un total de 31 ítems consistentes en preguntas con una escala de respuesta tipo Likert que va de 0 (ningún problema) a 3 (muchas dificultades). Con el PAIS-SR pueden obtenerse puntuaciones específicas para cada una de las subescalas, de manera que cuanto más elevada sea la puntuación de un sujeto, peor será su ajuste psicosocial en el ámbito correspondiente. El PAIS-SR se ha empleado en otras enfermedades, tales como el cáncer (Gumus, Cam y Malak, 2011), la diabetes (Akca y Cinar, 2008) y también los TCA (Quiles y Terol, 2011). En el presente estudio, las escalas mostraron una consistencia interna entre .81 y .93 .

\section{Malestar psicológico}

El malestar psicológico de los pacientes se midió con la Escala de Ansiedad y Depresión Hospitalaria (HADS; Zigmond y Snaith, 1983) en su versión española (Tejero, Guimera, Farré y Peri, 1986), y con el General Health Questionnaire-12 (GHQ-12; Goldberg y Williams, 1988), también adaptado al español (Rocha, Pérez, Rodríguez-Sans, Borrell y Obiols, 2011).

La HADS es una escala autoaplicada que detecta sintomatología psicológica de depresión y ansiedad mediante 14 ítems. Está compuesta por dos subescalas, Ansiedad y Depresión, de siete ítems cada una con una escala de respuesta tipo Likert de cuatro puntos, de manera que a mayor puntuación en cada subescala, mayor nivel de ansiedad y/o depresión. Este instrumento ha sido aplicado en poblaciones con diferentes patologías tales como esquizofrenia o fibromialgia (Allan y Martin, 2009; Pastor, Lledó, López-Roig, Pons y Martín-Aragón, 2010), y posee propiedades psicométricas adecuadas, con una consistencia interna de las subescalas entre .80 y .86 (Quintana et al., 2003). En este estudio, la consistencia interna fue de .82 para la subescala de ansiedad y .89 para la de depresión.

El GHQ-12 es un cuestionario autoadministrado de cribado para detectar morbilidad psicológica mediante la evaluación del estado durante el mes anterior. Está compuesto por 12 ítems, 6 de ellos directos y los otros 6 inversos, con una escala de respuesta tipo Likert de cuatro puntos (de 0 a 3 ). Puede ser empleado como una escala que evalúa un único factor, siendo recomendado para su corrección el uso de la puntuación GHQ, por ser la más adecuada cuando el instrumento se considera unidimensional (Rocha, Pérez, RodríguezSans, Borrell y Obiols, 2011). Para este tipo de puntuación, de las cuatro opciones de respuesta, las dos primeras se puntúan con 0 , mientras que las dos últimas se puntúan con 1 , de manera que el valor máximo obtenido en el cuestionario es de 12 y el menor es de 0 . Las propiedades psicométricas de este instrumento para población española señalan una

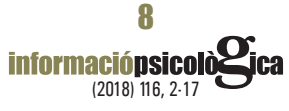


consistencia interna de .86 , en el estudio llevado a cabo por los autores mencionados anteriormente. En este trabajo, se obtuvo un alfa de Cronbach de .93.

\section{Procedimiento}

Junto con los responsables de la Unidad de Trastornos Alimentarios y de la Unidad de Salud Mental, se envió una carta invitando a participar voluntariamente en esta investigación a todos los pacientes que habían recibido atención en estos centros en el último año. En ella se les informó de los objetivos y de la confidencialidad de los datos, y se les adjuntó en el sobre una batería de pruebas autoinformadas, con instrucciones concretas sobre su cumplimentación. Posteriormente, aquellos que aceptaron la participación en el estudio, entregaron de nuevo en estos centros las pruebas completas y el consentimiento informado. En el caso de los pacientes menores de edad, fueron sus responsables legales quienes firmaron el consentimiento.

\section{Análisis}

Los análisis estadísticos se realizaron con el programa IBM SPSS v.20. Se obtuvieron las frecuencias y los descriptivos de los datos en cuanto a media, desviación típica, mediana, mínimo y máximo. Las relaciones existentes entre las dimensiones de la representación se comprobaron mediante la correlación de Spearman. Por último, la relación entre la representación de la enfermedad y las distintas áreas del ajuste psicosocial o el malestar psicológico se analizó a través de la regresión múltiple mediante el procedimiento de pasos sucesivos (stepwise).

\section{Resultados}

Representación de la enfermedad

En la Tabla 2 se presentan los estadísticos descriptivos de las variables relativas a las dimensiones de la representación de la enfermedad en los pacientes de TCA. Como se puede observar, los participantes presentaron altas puntuaciones en las dimensiones consecuencias,

Tabla 2.- Descriptivos de las dimensiones de la representación de la enfermedad en las personas con el TCA.

\begin{tabular}{lccccc}
\hline & Media & DT & Mediana & Mínimo & Máximo \\
\hline Consecuencias & 7.04 & 2.57 & 8.00 & 1.00 & 10.00 \\
Duración & 5.56 & 2.85 & 5.00 & 1.00 & 10.00 \\
Control & 4.80 & 2.89 & 5.00 & 0.00 & 10.00 \\
Curación & 6.62 & 2.64 & 7.00 & 0.00 & 10.00 \\
Identidad & 6.11 & 2.75 & 6.00 & 0.00 & 10.00 \\
Preocupación & 6.57 & 2.94 & 7.00 & 0.00 & 10.00 \\
Coherencia & 7.66 & 2.78 & 8.50 & 0.00 & 10.00 \\
Representación emocional & 7.32 & 2.80 & 8.00 & 1.00 & 10.00 \\
\hline
\end{tabular}


coherencia y representación emocional. Estos resultados indican que los pacientes con un TCA percibieron su enfermedad con consecuencias serias, entendían lo que les sucedía y experimentaron reacciones emocionales negativas asociadas a su situación. También obtuvieron puntuaciones superiores al punto medio del rango en las dimensiones duración, curación, identidad y preocupación. Por otro lado, los pacientes obtuvieron las puntuaciones más bajas en la dimensión control, lo cual indica que no percibieron un control personal elevado sobre la enfermedad.

\section{Relaciones entre las dimensiones de la representación}

Se examinaron las correlaciones entre las dimensiones de la representación de la enfermedad, mostradas en la Tabla 3. La dimensión consecuencias presentó relaciones significativas y en sentido positivo con las dimensiones identidad, preocupación y representación emocional, y en sentido negativo con la dimensión control. La dimensión duración mostró relaciones significativas y en sentido positivo con la representación emocional y la identidad, y relaciones en sentido negativo con la percepción de control y de curación. La dimensión control presentó una relación positiva y significativa con la percepción de curación, y negativa con las dimensiones identidad, preocupación y representación emocional. Además, aparecieron relaciones significativas y en sentido positivo de la identidad con las dimensiones preocupación y representación emocional. Por último, la dimensión preocupación presentó una relación significativa y en sentido positivo con la representación emocional.

Tabla 3.- Relaciones entre las dimensiones de la representación de la enfermedad en las personas con el TCA

\begin{tabular}{lcccccccc}
\hline & Consec & Durac & Contr & Curac & Ident & Preoc & Coher & Emoc \\
\hline Consec & 1 & .191 & $-.353^{* *}$ & -.001 & $.673^{* *}$ & $.665^{* *}$ & .052 & $.702^{* *}$ \\
Durac & - & 1 & $-.348^{* *}$ & $-.368^{* *}$ & $.295^{*}$ & .171 & .037 & $.342^{*}$ \\
Contr & - & - & 1 & $.450^{* *}$ & $-.505^{* *}$ & $-.314^{*}$ & .192 & $-.474^{* *}$ \\
Curac & - & - & - & 1 & -.178 & .026 & .140 & -.095 \\
Ident & - & - & - & - & 1 & $.552^{* *}$ & -.031 & $.650^{* *}$ \\
Preoc & - & - & - & - & - & 1 & .144 & $.659^{* *}$ \\
Coher & - & - & - & - & - & - & 1 & -.007 \\
Emoc & - & - & - & - & - & - & - & 1 \\
\hline
\end{tabular}

Consec: Consecuencias; Durac: Duración; Contr: Control; Curac: Curación; Ident: Identidad; Preoc: Preocupación; Coher: Coherencia; Emoc: Emociones; *p <.05; **p <.01

\section{Representación de la enfermedad, ajuste psicosocial y malestar psicológico}

Los resultados del análisis de regresión múltiple se muestran en la Tabla 4. El ajuste psicosocial incluyó las cinco subescalas del PAIS-SR seleccionadas. Se observa que las dimensiones consecuencias y control fueron las que más contribuyeron en la explicación de las varianzas de las distintas áreas del ajuste psicosocial, en concreto en el ámbito profesional/ 
Tabla 4.- Regresión múltiple mediante pasos sucesivos: Representación de la enfermedad, ajuste psicosocial y malestar psicológico

\begin{tabular}{|c|c|c|c|c|}
\hline Variable dependiente / Predictores & $\mathrm{R}^{2} / \mathrm{r}$ & Cambio en $\mathrm{R}^{2}$ & $\mathrm{~F}$ & $\beta$ \\
\hline Ajuste psicosocial / Representación enfermedad & & & & \\
\hline Ámbito profesional/académico & $.28 / .53$ & & $17.72 * * *$ & $.53 * * *$ \\
\hline Consecuencias & & .28 & & \\
\hline Ámbito doméstico & $.41 / .64$ & & $8.85^{* *}$ & \\
\hline Consecuencias & & .25 & & $.46^{* *}$ \\
\hline Control & & .16 & & $-.41 *$ \\
\hline Relaciones sexuales & $.29 / .54$ & & $8.01 *$ & $-.54 *$ \\
\hline Control & & .29 & & \\
\hline Relaciones familiares & $.16 / .40$ & & $8.81 * *$ & $.40^{* *}$ \\
\hline Identidad & & .16 & & \\
\hline Ámbito social & $.33 / .58$ & & $12.05 * * *$ & \\
\hline Preocupación & & .27 & & $.34 *$ \\
\hline Representación emocional & & .06 & & $.31 *$ \\
\hline \multicolumn{5}{|l|}{ Malestar psicológico / Representación enfermedad } \\
\hline Ansiedad & $.49 / .69$ & & $21.49 * * *$ & \\
\hline Representación emocional & & .32 & & $.43 * * *$ \\
\hline Duración & & .16 & & $.42 * * *$ \\
\hline Depresión & $.23 / .48$ & & $15.12 * * *$ & \\
\hline Representación emocional & & .23 & & $.48^{* * *}$ \\
\hline Distrés & $.42 / .65$ & & $21.60 * * *$ & \\
\hline Control & & .32 & & $-.40 * *$ \\
\hline Representación emocional & & .10 & & $.36^{* *}$ \\
\hline
\end{tabular}

$* \mathrm{p}<.05 ; * * \mathrm{p}<.01 ; * * * \mathrm{p}<.001$

académico, el doméstico y las relaciones sexuales. En este sentido, aquellos pacientes que percibieron mayores consecuencias para la vida, tuvieron un peor ajuste en los ámbitos profesional/académico y en el doméstico, mientras que una alta percepción de control se relacionó con un ajuste más positivo en el ámbito doméstico y en las relaciones sexuales. La identidad sólo fue significativa en el ámbito de las relaciones familiares, explicando un 16\% de la varianza. En este caso, una mayor identidad con el problema se asoció a un peor ajuste 
respecto al área familiar. Por otro lado, en referencia al ámbito social, fueron las dos dimensiones relacionadas con la representación emocional las más significativas, explicando un $33 \%$ de la varianza entre ambas en el ámbito mencionado. La dimensión curación no apareció como variable predictora significativa en ninguno de los ámbitos del ajuste psicosocial.

En cuanto al malestar psicológico, incluyó las escalas de ansiedad y depresión del HADS y la puntuación total del GHQ-12, denominado como distrés. La representación emocional fue la dimensión más significativa en relación al malestar psicológico, explicando un 32\% de la varianza de la ansiedad, un $23 \%$ de la depresión y un $10 \%$ de distrés. Además, las creencias de duración del problema contribuyeron a explicar la varianza de la ansiedad en un $16 \%$ y el control explicó un 32\% de la varianza del distrés. En esta línea, la representación emocional más negativa y la creencia de que la enfermedad duraría mucho tiempo, se relacionó en sentido positivo con sintomatología de ansiedad, depresión y distrés psicológico. Sin embargo, para esta última variable se obtuvo una relación en sentido negativo con la percepción de control.

\section{Discusión}

Este trabajo trata de describir la representación de la enfermedad en personas afectadas por un TCA y las relaciones entre sus dimensiones, y evaluar la relación de las dimensiones de la representación con el ajuste psicosocial y el malestar psicológico.

En cuanto a la representación de la enfermedad, en el presente estudio los pacientes percibieron consecuencias serias para su vida. Ello concuerda con los resultados de otros estudios, en los que la mayoría de afectados de TCA también consideraron repercusiones graves para el funcionamiento general (DeJong et al., 2012; Holliday et al., 2005; Quiles et al., 2007). Los pacientes mostraron también puntuaciones medias en cuanto a la identidad con la enfermedad y tenían la creencia de que podían curarse mediante el tratamiento. Este último resultado puede explicarse si se considera que todos ellos seguían terapia psicológica en el momento en que fueron evaluados. Estos hallazgos son congruentes con los de Quiles et al., pero difieren de los resultados de Holliday et al. debido a que en este último estudio los participantes no estaban sometidos a ningún tipo de tratamiento. Del mismo modo, respecto a las creencias de curación, los profesionales que ayudan a los afectados suelen hacer hincapié en los beneficios de experimentar un proceso terapéutico guiado.

Por último, los pacientes presentaron puntuaciones cercanas al punto medio del rango en la percepción de duración de la enfermedad y puntuaciones bajas en relación a la creencia de control. Ello se diferencia de los resultados encontrados en el estudio de Holliday et al. (2005), en el que los afectados consideraron su situación como crónica y sin posibilidad de control por su parte, y de los hallazgos de Quiles et al. (2007), cuyos pacientes, a pesar de creer que el problema era crónico, percibieron un alto control sobre la situación. En este estudio, ambas creencias pueden deberse a que, desde el enfoque cognitivo-conductual del que parte el tratamiento que los afectados seguían en el momento de la evaluación, se favorece la percepción de control sobre el problema y de capacidad para evitar que se cronifique. No obstante, los profesionales también suelen advertir al paciente sobre la necesidad de mantenerse alerta respecto a las posibles recaídas en la enfermedad, ya que en los TCA son 
numerosos los factores que pueden desencadenar un empeoramiento de la situación. Es por ello que los participantes pudieron obtener puntuaciones medias en la percepción de duración del problema y algo más bajas en la percepción de control.

En cuanto a las relaciones entre las dimensiones de la representación de la enfermedad, la primera hipótesis de partida fue que la identidad presentaría relaciones positivas y significativas con la percepción de duración y con la dimensión consecuencias. Los resultados hallados permitieron confirmar esta hipótesis, debido a que los pacientes que presentaron mayor identidad con el problema tendieron a considerar que éste tenía consecuencias serias para su funcionamiento y una duración mayor. La segunda hipótesis fue que la identidad presentaría relaciones negativas y significativas con la percepción de control y de curación. En este caso, los resultados no permitieron confirmar esta hipótesis debido a que, pese a que una identidad mayor con la enfermedad se asoció a una menor percepción de control sobre la situación, no se encontró la relación significativa esperada entre la identidad y la dimensión curación. En este sentido, el resultado puede ser atribuible nuevamente al tratamiento que siguen los pacientes dado que, específicamente para los TCA, uno de los puntos clave de la intervención es la descripción a los afectados de la sintomatología típica del trastorno, con tal de que conozcan sus características y tomen conciencia de la situación en la que se encuentran para poder actuar frente a ella. De este modo, una identificación elevada con la enfermedad no siempre está asociada a una débil creencia de que es posible mejorar mediante el tratamiento. Por otra parte, otros resultados hallados en el estudio señalan que la creencia de cronicidad del problema se relacionó en sentido negativo con la percepción de control y de curación mediante el tratamiento. Además, la representación emocional negativa se relacionó también en sentido negativo con la percepción de control, y en positivo con la de consecuencias serias, con la identificación con el problema y con la creencia de cronicidad. Por otro lado, la percepción de control sobre la situación se asoció con la creencia de curación mediante el tratamiento. Estos resultados son similares a los obtenidos por Quiles et al. (2007), en los que la representación emocional se relacionó de manera significativa y en sentido positivo con las dimensiones identidad, consecuencias y duración, y la percepción de control se asoció en sentido positivo y significativamente con la dimensión curación.

El último objetivo del presente trabajo fue analizar la relación entre las dimensiones de la representación de la enfermedad con el ajuste psicosocial y con el malestar psicológico. Los resultados no permitieron confirmar la hipótesis de partida, en la que se esperaba que la identidad, la duración y las consecuencias percibidas de la enfermedad se relacionarían en negativo con el ajuste psicosocial, y en positivo con el malestar psicológico, mientras que el control y la curación lo harían en sentido positivo con el ajuste psicosocial y en negativo con el malestar psicológico. Las dimensiones identidad, duración, consecuencias y control presentaron las relaciones esperadas, pero no ocurrió así en el caso de la dimensión curación. Concretamente, aquellos pacientes con una identidad más elevada con el problema tendieron a experimentar un peor ajuste en las relaciones familiares. En este sentido, una percepción elevada de síntomas por parte del paciente puede favorecer que espere reacciones de crítica y rechazo de sus familiares, siendo éste uno de los principales miedos del afectado, tal y como proponen Schmidt y Treasure (2006) en su modelo de mantenimiento 
cognitivo-interpersonal del TCA. Por ello, es posible que una identificación elevada con la enfermedad se relacione con una evitación mayor de las interacciones personales en el núcleo familiar. Otro de los resultados señaló que la creencia de consecuencias serias se asoció a un peor ajuste en los ámbitos profesional/académico y doméstico. Este resultado puede deberse a que el tratamiento de tipo cognitivo-conductual seguido por los pacientes fomenta que estos entiendan las repercusiones del TCA en los aspectos cognitivos, emocionales, físicos, conductuales y sociales. De esta manera, se intenta conseguir que los afectados vinculen las consecuencias de la enfermedad a esos niveles con los resultados negativos en los diferentes ámbitos vitales. Otra explicación alternativa es que un alto porcentaje de participantes precisaron uno o más ingresos hospitalarios para la recuperación orgánica en el pasado, lo cual pudo interrumpir las actividades en estos ámbitos y, por tanto, dificultar el ajuste.

Por otro lado, una percepción mayor de control sobre la situación se asoció a un ajuste más adecuado en el ámbito doméstico y en las relaciones sexuales. Respecto a este último aspecto, factores como la baja percepción de control, que puede entenderse como la baja percepción de autoeficacia frente al problema, se asocian con una pérdida de libido y un aumento de la ansiedad sexual en personas con un TCA (Poyastro, et al., 2010). Respecto al ámbito social, fue la representación emocional negativa la que se relacionó con un peor ajuste. En este aspecto, la expresión de emociones suele ser percibida por los pacientes de TCA como un signo de debilidad y vulnerabilidad frente a los otros significativos, lo cual fomenta el aislamiento y la evitación de las interacciones sociales cuando los afectados experimentan emociones negativas (Kyriacou, Easter y Tchanturia, 2009).

En lo referente a la relación de las dimensiones de la representación de la enfermedad con el malestar psicológico, una representación emocional más negativa se asoció con mayores niveles de depresión, ansiedad y distrés en general, lo cual coincide con los resultados hallados por Quiles et al. (2007). Además, las creencias de cronicidad de la enfermedad se relacionaron con un mayor nivel de ansiedad, lo que puede atribuirse a una percepción de indefensión frente a la creencia de que el problema se mantendrá a lo largo del tiempo. Por último, una mayor percepción de control se relacionó con un menor distrés psicológico. Como señalan Hagger y Orbell (2003), para los pacientes de enfermedades crónicas, la percepción de control se relaciona con la búsqueda de apoyo social, la expresión de emociones y las estrategias de afrontamiento centradas en el problema, lo que podría implicar un menor distrés.

El presente estudio cuenta con una serie de limitaciones. En primer lugar, el diseño de la investigación fue transversal, de manera que no pueden deducirse relaciones causales entre las variables partiendo de los resultados. Por otro lado, el tamaño muestral fue reducido y los distintos diagnósticos de TCA no estuvieron representados de manera equitativa, debido a la diferencia en el número de personas pertenecientes a cada categoría. Esto puede dificultar la extrapolación de resultados a otras muestras. Otra limitación consiste en que el uso del IPQ en su versión abreviada, aunque requiere menos tiempo para su cumplimentación, no cuantifica la percepción de duración cíclica en el paciente. 
A pesar de estas cuestiones, los hallazgos del presente trabajo sugieren la importancia de considerar las cogniciones individuales de los pacientes respecto a su problema de salud. Así, trabajar con ellos para reforzar la percepción de control personal y mostrar los beneficios del tratamiento, además de racionalizar los pensamientos relacionados con la cronicidad de la enfermedad, puede mejorar el ajuste psicosocial y disminuir las repercusiones negativas en el estado emocional. Asimismo, para los TCA las tasas de abandono del tratamiento psicológico son elevadas (Mahon, 2000). Por ello, evaluar las percepciones sobre la enfermedad puede contribuir a que los pacientes continúen con el tratamiento y obtengan mejores resultados en salud. Como vías de investigación futuras se propone la realización de estudios longitudinales, incluyendo el análisis de las estrategias de afrontamiento y del proceso de evaluación posterior a la obtención de resultados en salud, con el objetivo de describir el ciclo completo que propone el Modelo de Auto-Regulación. Se plantea también la inclusión de variables objetivas, como por ejemplo el estado orgánico en términos de índice de masa corporal, para analizar si existe relación con los resultados autoinformados por los pacientes en las variables analizadas. Por otro lado, se considera relevante profundizar en los factores causales atribuidos al problema, con el objetivo de reparar en las posibles relaciones con el resto de dimensiones de la representación y con el ajuste psicosocial y emocional.

\section{Bībliografía}

Akca, A. T. \& Cinar, S. (2008). Comparison of psychosocial adjustment in people with diabetes with and without diabetic foot ulceration. Australian Journal of Advanced Nursing, 25(4), 87-96.

Allan, R. \& Martin, C. R. (2009). Can the Hospital Anxiety and Depression Scale be used in patients with schizophrenia? Journal of Evaluation in Clinical Practice, 15(1), 134-141.

Broadbent, E., Kydd, R., Sanders, D. \& Vanderpyl, J. (2008). Unmet needs and treatment seeking in high users of mental health services: Role of illness perceptions. Australian and New Zealand Journal of Psychiatry, 42(2), $147-153$.

Broadbent, E., Petrie, K. J., Main, J. \& Weinman, J. (2006). The Brief Illness Perception Questionnaire. Journal of Psychosomatic Research, 6O(6), 631-637.

Cowdrey, F. A. \& Park, R. J. (2012). The role of experiential avoidance, rumination and mindfulness in eating disorders. Eating Behaviors, 13(2), 100-105.

DeJong, H., Hillcoat, J., Perkins, S., Grover, M. \& Schmidt, U. (2012). Illness perception in bulimia nervosa. Journal of Health Psychology, 17(3), 399-408.

Derogatis, L. R. \& López, M. C. (1983). The psychosocial adjustment to illness scale (PAIS \& PAIS-SR): Administration, scoring and procedures manual-I. Baltimore, MD: Johns Hopkins University School of Medicine.

Dickson, A., Toft, A. \& O'Carroll, R. E. (2009). Neuropsychological functioning, illness perception, mood and quality of life in chronic fatigue syndrome, autoimmune thyroid disease and healthy participants. Psychological Medicine, 39(9), 1567-1576.

Evans, D. \& Norman, P. (2009). Illness representations, coping and psychological adjustment to Parkinson's disease. Psychology \& Health, 24(10), 1181-1196.

Fortune, G., Barrowclough, C. \& Lobban, F. (2004). Illness representations in depression. British Journal of Clinical Psychology, 43(4), 347-364. 
Godoy-Izquierdo, D., López-Chicheri, I., López-Torrecillas, F., Vélez, M. \& Godoy, J. F. (2007). Contents of lay illness models dimensions for physical and mental diseases and implications for health professionals. Patient Education and Counseling, 67(1), 196-213.

Goldberg, D. P. \& Williams, P. (1988). A user's guide to the General Health Questionnaire. Windsor: NFER-Nelson.

Gumus, A. B., Cam, 0. \& Malak, A. T. (2011). Relationships between psychosocial adjustment and hopelessness in women with breast cancer. Asian Pacific Journal of Cancer Prevention, 12(2), 433-438.

Hagger, M. \& Orbell, S. (2003). A meta-analytic review of the Common-Sense Model of illness representations. Psychology \& Health, 18(2), 141-184.

Harman, G. \& Clare, L. (2006). Illness representations and lived experience in early-stage dementia. Qualitative Health Research, 16(4), 484-502.

Holliday, J., Wall, E., Treasure, J. \& Weinman, J. (2005). Perception of illness in individuals with anorexia nervosa: a comparison with lay men and women. International Journal of Eating Disorders, 37(1), 50-56.

Kyriacou, 0., Easter, A. \& Tchanturia, J. (2009). Comparing views of patients, parents and clinicians on emotions in anorexia: A qualitative study. Journal of Health Psychology, 14(7), 843-854.

Lau, R. R. \& Hartman, K. A. (1983). Common sense representations of common illnesses. Health Psychology, 2(2), 165-187.

Leventhal, H., Diefenbach, M. \& Leventhal, E. A. (1992). Illness cognition: Using common sense to understand treatment adherence and affect cognition interactions. Cognitive Therapy and Research, 16(2), 143-163.

Leventhal, H., Meyer, D. \& Nerenz, D. (1980). The common sense representations of illness danger. En S. Rachman (Ed.), Contributions to medical psychology (pp. 60-78). Oxford: Pergamon Press.

Mahon, J. (2000). Dropping out from psychological treatment for eating disorders: What are the issues? European Eating Disorders Review, 8(3), 198-216.

Moss-Morris, R., Weinman, J., Petrie, K. J., Horne, R., Cameron, L. D. \& Buick, D. (2002). The Revised Illness Perception Questionnaire (IP0-R). Psychology and Health, 17(1), 1-16.

Neipp, M. C. (2005). Creencias de control y afrontamiento en mujeres con cácer de mama en la etapa de seguimiento. Tesis doctoral no publicada. Universidad Miguel Hernández, Alicante.

Pacheco-Huergo, V. (2007). Validación de un cuestionario de percepción de enfermedad: Illness Perception Questionnaire Revised (IPQ-R) and Brief Illness Perception Questionnaire (BIPQ). Proyecto financiado por el Fondo de Investigación Sanitaria del Instituto de Salud Carlos III (FIS PI031649).

Pastor, M. A., Lledó, A., López-Roig, S., Pons, N. \& Martín-Aragón, M. (2010). Predictores de la utilización de recursos sanitarios en la fibromialgia. Psicothema, 22(4), 549-555.

Poyastro, A., Raney, T.J., Thornton, L. M., Fichter, M. M., Berrettini, W. H., Goldman, D., ...Bulik, C. M. (2010). Sexual functioning in women with eating disorders. International Journal of Eating Disorders, 43(2), 123-129.

Quiles, Y. \& Terol, M. C. (2011). Using Common Sense Model in eating disorders. Journal of Health Psychology, 16(2), 208-216.

Quiles, Y., Terol, M. C., Romero, C. \& Pagán, G. (2007). Illness perception in eating disorders and psychosocial adaptation. European Eating Disorders Review, 15(5), 373-384.

Quintana, J. M., Padierna, A., Esteban, C., Arostegui, I., Bilbao, A. \& Ruiz, I. (2003). Evaluation of the psychometric characteristics of the Spanish version of the Hospital Anxiety and Depression Scale. Acta Psychiatrica Scandinavica, 107(3), 216-221.

Roberts, M. E., Tchanturia, K. \& Treasure, J. L. (2010). Exploring the neurocognitive signature of poor-shifting in anorexia and bulimia nervosa. Journal of Psychiatric Research, 44(14), 964-970.

Rocha, K. B., Pérez, K., Rodríguez-Sans, M., Borrell, C. \& Obiols, J. E. (2011). Propiedades psicométricas y valores normativos del General-Health Questionnaire (GH0-12) en población general española. International Journal of Clinical and Health Psychology, 11(1), 125-139.

Sassaroli, S., Gallucci, M. \& Ruggiero, G. M. (2008). Low perception of control as a cognitive factor of eating disorders. Its independent effects on measures of eating disorders and its interactive effects with perfectionism and sel- 
esteem. Journal of Behavior Therapy and Experimental Psychiatry, 39(4), 467-488.

Schmidt, U. \& Treasure, J. (2006). Anorexia nervosa: valued and visible. A cognitive-interpersonal maintenance model and its implications for research and practice. British Journal of Clinical Psychology, 45(3), 343-366.

Sensky, T. (1990). Patient's reactions to illness. British Medical Journal, 300(6725), 622-623.

Stockford, K., Turner, H. \& Cooper, M. (2007). Illness perception and its relationship to readiness to change in eating disorders: a preliminary investigation. British Journal of Clinical Psychology, 46(2), 139-154.

Tejero, A., Guimera, E. M., Farré, J. M. \& Peri, J. M. (1986). Uso clínico del HAD (Hospital Anxiety and Depression Scale) en población psiquiátrica: un estudio de su sensibilidad, fiabilidad y validez. Revista del Departamento de Psiquiatría de la Facultad de Medicina de Barcelona, 13(5), 233-238.

Zigmond, A. S. \& Snaith, R. P. (1983). The Hospital Anxiety and Depression Scale. Acta Psychiatrica Scandinavica, 67(6), 361-370. 